\title{
American College of Medical Genetics and Genomics Standards and Guidelines for Clinical Genetics Laboratories, 2014 edition: technical standards and guidelines for Huntington disease
}

\author{
Lora Bean, $\mathrm{PhD}^{1}$ and Pinar Bayrak-Toydemir, MD, PhD²; on behalf of the ACMG Laboratory Quality \\ Assurance Committee
}

\begin{abstract}
Disclaimer: These ACMG Standards and Guidelines are developed primarily as an educational resource for clinical laboratory geneticists to help them provide quality clinical laboratory genetic services. Adherence to these standards and guidelines is voluntary and does not necessarily ensure a successful medical outcome. These standards and guidelines should not be considered inclusive of all proper procedures and tests or exclusive of other procedures and tests that are reasonably directed toward obtaining the same results. In determining the propriety of any specific procedure or test, the clinical laboratory geneticist should apply his or her own professional judgment to the specific circumstances presented by the individual patient or specimen. Clinical laboratory geneticists are encouraged to document in the patient's record the rationale for the use of a particular procedure or test, whether or not it is in conformance with these standards and guidelines. They also are advised to take notice of the date any particular guideline was adopted, and to consider other relevant medical and scientific information that becomes available after that date. It also would be prudent to consider whether intellectual property interests may restrict the performance of certain tests and other procedures.
\end{abstract}

Huntington disease is an autosomal-dominant neurodegenerative disease of mid-life onset caused by expansion of a polymorphic trinucleotide $(\mathrm{CAG})$ repeat. Variable penetrance for alleles carrying 36-39 repeats has been noted, but the disease appears fully penetrant when the repeat numbers are $>40$. An abnormal CAG repeat may expand, contract, or be stably transmitted when passed from parent to child. Assays used to diagnose Huntington disease must be optimized to ensure the accurate and unambiguous quantitation of CAG repeat length. This document provides an overview of Huntington disease and methodological considerations for Huntington disease testing. Examples of laboratory reports are also included.

Genet Med advance online publication 30 October 2014

Key Words: clinical testing; Huntington disease; polyglutamine; practice guideline; trinucleotide repeat disorder
Disease-specific statements are intended to augment the general American College of Medical Genetics and Genomics (ACMG) Standards and Guidelines for Clinical Genetics Laboratories. Individual laboratories are responsible for meeting the Clinical Laboratory Improvement Amendments (CLIA)/College of American Pathologists (CAP) quality assurance standards with respect to appropriate sample documentation, assay validation, general proficiency, and quality control measures. This 2014 edition of the guideline updates and supersedes the original laboratory guideline on this topic, which was published in $2004^{1}$ and reaffirmed once during the ensuing decade.

\section{BACKGROUND OF HUNTINGTON DISEASE Gene symbol/protein name \\ HTT (historically known as IT15)/Huntingtin. The GenBank accession number for the reference sequence is NM_002111. The OMIM gene/locus code is 613004 .}

\section{OMIM phenotype number}

143100.

\section{Brief clinical description}

Huntington disease (HD) is a neurodegenerative disease of mid-life onset that produces choreic movements and cognitive decline, often accompanied by psychiatric changes. It affects 3-5 out of 100,000 individuals. However, the prevalence of HD exceeds 15 per 100,000 in some populations, mostly of western European origin. ${ }^{2}$ Juvenile-onset HD occurs in approximately $5 \%$ of affected patients, is a rapidly progressive variant, and presents with rigidity, spasticity, and intellectual decline before the age of 20 years. The symptoms result from the selective loss of neurons, most notably in the caudate nucleus and putamen, and there is currently no effective treatment. For more information, see the online Gene Reviews profile at http://www.ncbi. nlm.nih.gov/books/NBK1305/.

${ }^{1}$ Department of Human Genetics, Emory University School of Medicine, Atlanta, Georgia, USA; ${ }^{2}$ Department of Pathology, University of Utah School of Medicine and ARUP

Laboratories, Salt Lake City, Utah, USA. Correspondence: Pinar Bayrak-Toydemir (pinar.bayrak-toydemir@aruplab.com) 


\section{Mode of inheritance}

Inheritance is autosomal dominant, with clinical manifestations associated with expansion of a polymorphic trinucleotide (CAG) repeat. Variable penetrance for alleles carrying 36-39 repeats has been noted, but the disease appears fully penetrant when the repeat numbers are $\geq 40$.

An abnormal CAG repeat may expand, contract, or be stably transmitted when passed from parent to child. Although small expansions and contractions are common in maternal and paternal transmission of abnormal alleles, large expansions occur only in paternal transmissions. ${ }^{3}$

\section{Gene description/normal gene product}

The HTT gene on $4 \mathrm{p} 16.3$ spans $170 \mathrm{~kb}$ and contains 67 exons. The encoded protein, huntingtin, has 3,144 amino acid residues, has a molecular weight of $350 \mathrm{kDa}$, and lacks homology to any previous known proteins. HD is caused by an expansion of a highly polymorphic CAG repeat in exon 1 of the HTT gene. The expanded CAG repeat is translated into a polyglutamine tract in the huntingtin protein. Structural analysis of the HTT gene promoter region is consistent with the gene being a housekeeping gene. Although on a cellular level mutant huntingtin is widely expressed in both neural and non-neural tissue, there is region-specific neuronal loss in the neurons in the caudate and putamen.

\section{Genotype/phenotype association}

The inverse relationship between the CAG repeat size (at $\geq 40$ repeats) and age of onset is unequivocal. The relationship is particularly strong for high repeat sizes ( $>50$ repeats). However, the repeat length does not allow precise prediction of the age of onset for any one particular patient.

\section{Mutational mechanism}

The abnormal polyglutamine expansion results in protein misfolding, nuclear accumulation and aggregation of HTT fragments, and altered post-translational modifications. ${ }^{4}$ Intranuclear inclusions of the truncated mutant protein aggregates have been identified in brains of HD patients. These alterations are ultimately associated with, but not necessarily causative of, cell death.

\section{Listing of mutations}

Allelic heterogeneity has not been described in HD.

\section{Ethnic association of HD mutation}

All major ethnic groups appear to be susceptible to expansion of the HD CAG repeat.

\section{SPECIAL TESTING CONSIDERATIONS \\ Clinical sensitivity and specificity \\ CAG repeat expansion mutations account for $>99 \%$ of cases of HD. Therefore, tests that effectively detect and measure the CAG repeat region of the HTT gene are $>99 \%$ sensitive. The absence of HD pathology has not been documented in any}

individual with an HD allele size of $\geq 40$ CAG repeats who died, disease free, after living up to or past the normal life expectancy. Therefore, positive results (at least one allele of 40 CAG repeats or more) are $100 \%$ specific. Allele sizes of 26 CAG repeats or less have never been associated with an HD phenotype in the US survey or in any published study. Allele sizes of 27-35 CAG repeats are rare and have not been associated convincingly with an HD phenotype but they may be mutable, as described in the Mutable Normal Alleles section. Allele sizes of 36-39 CAG repeats have been reported in both clinically affected and clinically unaffected individuals. Thus, it is not possible to determine the specificity of a test when one or both of the alleles are in the CAG repeat range of 36-39. CAG repeat expansion is offered as a clinical test. ${ }^{1}$

\section{Diagnostic versus predictive testing}

Detection of CAG expansion is used for both confirmatory and predictive testing. Positive results for both confirmatory and predictive testing are considered diagnostic. It is strongly suggested that predictive testing not be offered to individuals until they are at least 18 years old. A formal multidisciplinary predictive testing protocol is offered at many sites for individuals desiring determination of their carrier status.

\section{Prenatal testing}

Prenatal testing can be used for prenatal diagnosis in amniotic fluid cells and in chorionic villus samples. Maternal cell contamination studies should be performed on every prenatal sample to confirm the fetal origin of the sample being tested. Evidence of a molecular diagnosis of HD in the family should be obtained before prenatal testing.

\section{GUIDELINES}

\section{Definition of normal and mutation category}

Normal alleles. Normal alleles are defined as alleles with $\leq 26$ CAG repeats. ${ }^{5-7}$ These alleles are not pathologic and segregate as a stable polymorphic repeat in $>99 \%$ of meiosis (Figure 1).

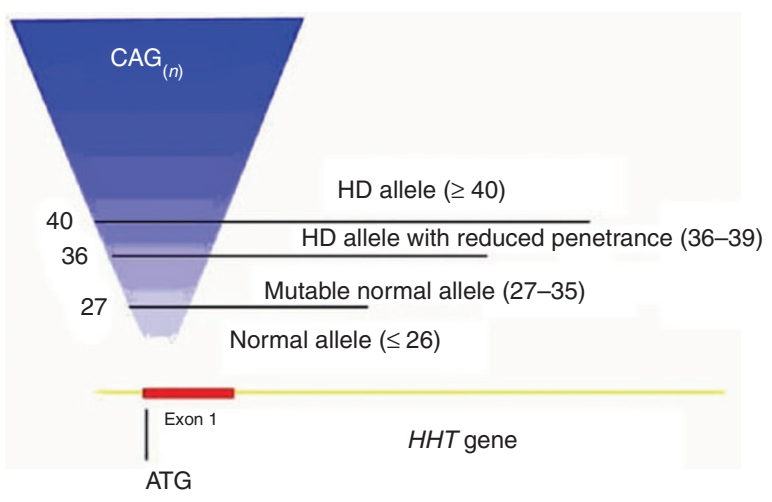

Figure 1 Diagram of $H T T$ gene and the location of the polymorphic CAG repeat within exon 1. Boundaries denote CAG repeat length categories and descriptors. 
The most common normal allele lengths contain 17 and 19 CAG repeats. ${ }^{5}$

Mutable normal alleles. Mutable normal alleles are defined as alleles with 27-35 CAG repeats, and this repeat range is often referred to as the meiotic instability range, or "intermediate alleles." These alleles have yet to be convincingly associated with an HD phenotype, but they can be meiotically unstable in sperm, and pathologic expansion of paternally derived alleles in this size range has been described. ${ }^{8,9}$ There have been no reports of maternally transmitted alleles in this range producing offspring with affected alleles..$^{10}$ Approximately $1.5-2 \%$ of the general population carry alleles in this size range. ${ }^{6,11}$ The likelihood that transmission of an allele in this range will expand into an HD allele is dependent on several factors, including the sex of the transmitting individual, the size of the allele, the molecular configuration of the region surrounding the CAG repeat, and its haplotype. ${ }^{11,12}$ This risk may be as high as $6-10 \%$ for paternal alleles carrying a CAG repeat of $35 .{ }^{12}$

$H D$ alleles with reduced penetrance. HD alleles with reduced penetrance are defined as alleles with 36-39 CAG repeats. Repeat sizes in this range are often referred to as being in the reduced penetrance range. Alleles in this size range are meiotically unstable and are associated with the HD phenotype in both clinically and neuropathologically documented cases. ${ }^{7}$ However, in rare cases, alleles in this range have been found in elderly asymptomatic individuals. ${ }^{12,13}$ Although the limited number of documented cases has precluded the development of empirical penetrance risks for alleles in this range, some estimates of risk can be ascertained from an examination of individual case reports. ${ }^{13,14}$

$H D$ alleles with full penetrance. HD alleles with full penetrance are defined as alleles with $\geq 40 \mathrm{CAG}$ repeats. Although there are no reports of the absence of HD pathology in any individual with $\geq 40$ CAG repeats, a few clinically asymptomatic carriers with 40 and 41 CAG repeats have been reported. ${ }^{14}$ The largest expanded HD allele detected to date carries $\sim 250$ CAG repeats in a patient with juvenile-onset disease. ${ }^{15}$

Mosaicism. Mosaicism attributable to both mitotic and meiotic instability has been described in brain and sperm, and it appears to be more pronounced in juvenile-onset cases of $\mathrm{HD}$ associated with larger CAG expansions. ${ }^{16}$ However, the degree of mosaicism is not significant enough to compromise the interpretation of CAG repeat lengths determined from DNA extracted from peripheral blood lymphocytes.

\section{Methodological considerations}

Individual US laboratories offering molecular diagnostic testing for HD should be in compliance with all federal and state regulations relevant to clinical laboratory operations. This includes meeting all CLIA/CAP quality control requirements. In addition, all US laboratories offering clinical testing for HD should be active participants in semi-annual HD proficiency testing challenges. All methodological applications should also be in compliance with the Standards and Guidelines for Clinical Genetics Laboratories developed by the ACMG. ${ }^{17}$ Non-US laboratories should be similarly compliant with their individual countries' statutory regulations governing oversight of clinical laboratories.

PCR methods. Several sets of primers, polymerase chain reaction (PCR) conditions, and amplicon separation and detection techniques have been published. ${ }^{18-22}$ Regardless of the particular PCR-based strategy selected, it is important for assay conditions and post-PCR analyses to be optimized to ensure the accurate and unambiguous quantitation of repeat length (Figure 2a,b). It should be noted that CAG sizing anomalies have been observed in comparative post-PCR analyses utilizing agarose, capillary, and denaturing polyacrylamide gel electrophoretic methods. ${ }^{23-25}$ As such, accurate quantitation of patient amplicon sizes should be empirically determined by comparison with appropriate external or internal standards. These could include, but are not limited to, size standard, cloned reference standards, and appropriate normal and abnormal patient controls whose sizes have been independently verified. Because it is the length of the polymorphic CAG repeat alone that is associated with the HD phenotype, patient genotyping based on the use of a single primer pair that amplifies both the CAG and the adjacent CCG repeat ${ }^{22}$ is discouraged. The CCG repeat, which lies $3^{\prime}$ of the HD CAG repeat, has been shown to be polymorphic; as a result, it may lead to diagnostic inaccuracies for both normal and HD allele sizing.

Appropriate controls that include a range of CAG sizes should be utilized for each analysis. It is the responsibility of the laboratory to empirically determine the detection limits for their assays. Although the upper limit of detection is not known, alleles carrying $\sim 115$ CAG repeats have been amplified by PCRbased methods, ${ }^{26}$ whereas alleles carrying more than $~ 125$ CAG repeats appear to be refractory to reproducible amplification..$^{15}$

Polymorphisms surrounding or within the CAG tract have been identified and have a collective frequency of $>1 \%$ in patients referred for HD testing. ${ }^{11,12,27-31}$ These nucleotide substitutions generally can be categorized into two groups: those that modify primer-annealing sites and those that result in the loss of interruption between the CAG and CCG tracts. In the first category, nucleotide changes can result in the misinterpretation of genotyping data due to an allele-specific amplification failure associated with primer mis-annealing. As such, in certain clinical circumstances "apparent homozygosity" for two normal alleles identified with standard PCR methods should be interpreted with caution, and the use of alternative primer pairs, triplet repeat primed PCR, or other methodologies (Southern blot or sequencing) should be used to resolve any ambiguous results. ${ }^{27-31}$ In the second category, rare A-to-G substitutions in the intervening 12-bp segment between the CAG and CCG tracts (CAG CAG CCG CCG) can result in increased meiotic instability of the tract as well as a miscalculation of 
uninterrupted CAG repeat length based on the conventional calculation formula. ${ }^{12,30}$

Southern blot. If the method routinely used by the laboratory is not validated to detect large CAG expansions, then Southern blotting should be used for the identification of expansions associated with juvenile-onset $\mathrm{HD}$ in cases of apparently homozygous normal genotypes. ${ }^{32}$

Triplet-repeat primed PCR (TP PCR). The HD CAG ${ }_{(n)}$ repeat region is amplified by PCR using a fluorescently labeled forward primer, located upstream of the $\mathrm{CAG}_{(n)}$ region, and a chimeric reverse primer located partially within the $\mathrm{CAG}_{(n)}$ region (Figure $3 \mathbf{a}$ and primer sequences Figure $3 \mathbf{b}$ ). The chimeric reverse primer hybridizes to multiple locations within the $\mathrm{CAG}_{(n)}$ repeat region, creating PCR products of varying sizes. Reactions are separated by capillary electrophoresis. TP PCR provides a characteristic ladder on the fluorescence trace, enabling the rapid identification of large pathogenic repeats that cannot be amplified using flanking primers. Because the $5^{\prime}$ end of the reverse chimeric primer exactly matches the sequence $3^{\prime}$ of the $\mathrm{CAG}_{(n)}$ region, this product is preferentially amplified. The true alleles are therefore distinguished as the highest peaks; stutter peaks extending from the smaller allele and terminating with the larger allele represent products from the reverse primer hybridizing within the $\mathrm{CAG}_{(n)}$ region. When an allele is too large to be amplified by this assay, a stutter peak of decreasing amplitude extends to the end of the electropherogram (see Figure 4a-d for examples). Results are evaluated for the presence of this distinct stutter pattern extending into the expanded allele range. ${ }^{33}$ Positive control (large repeat), negative control (normal size repeat), and no template control should be included on each run. Post-PCR detection of the alleles can be achieved by fragment length analysis.

Although TP PCR can be used as a primary method, it can be used instead of Southern blot for the identification of large expansions associated with juvenile-onset HD and is useful for the confirmation of "homozygous normal" genotypes.
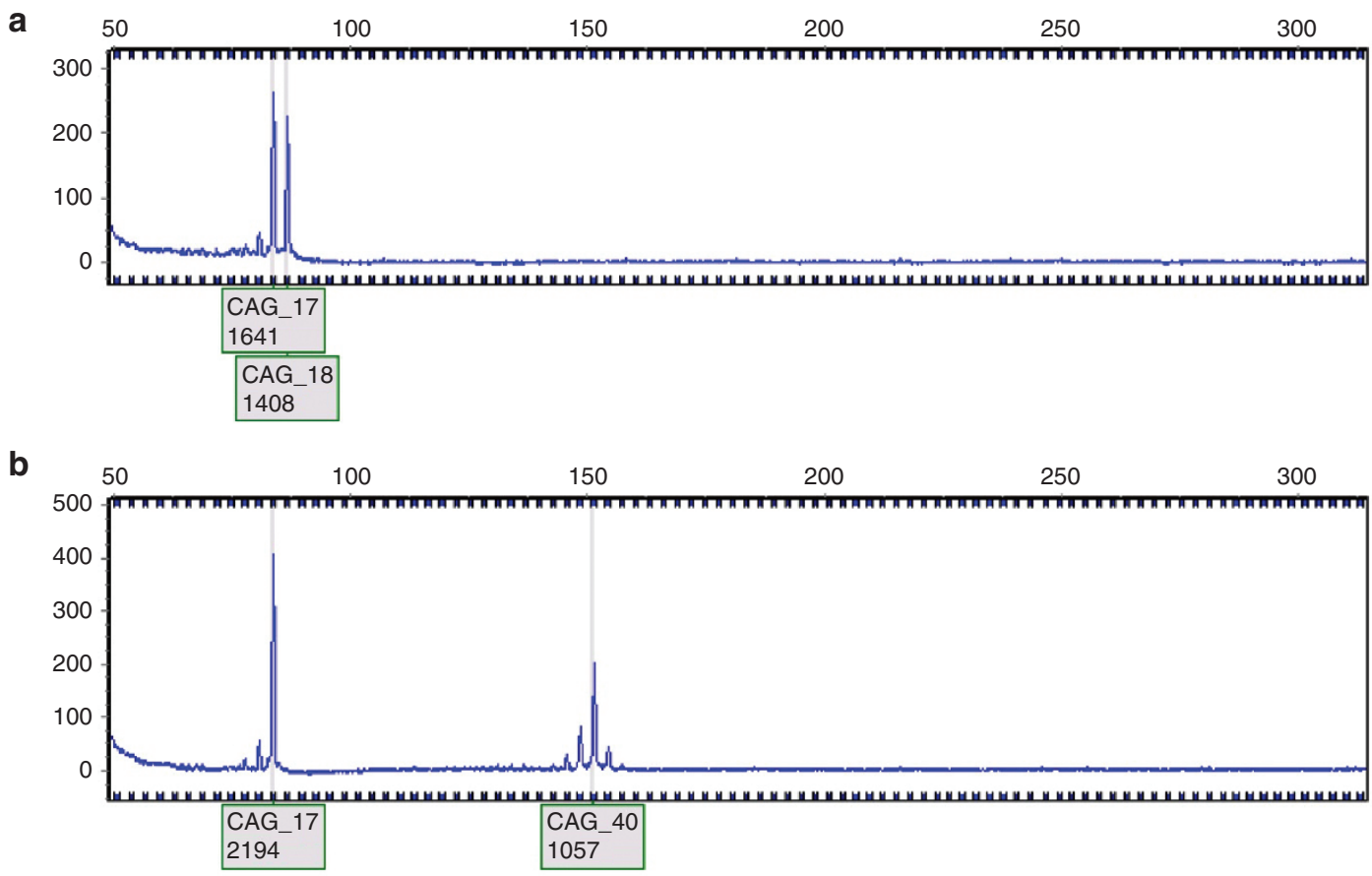

Figure 2 Polymerase chain reaction (PCR) results with different allele sizes. (a) PCR results with 17 and 18 CAG repeats. (b) PCR results with 17 and 40 CAG repeats.

a

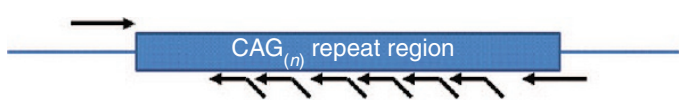

\section{b}

GATGAAGGCCTTCGAGTCCCTCAAGTCCTTCCAGCAGCAGCAGCAGCAGCAGCAGCAGCAGCAGCAGCAGCAGC
AGCAGCAGCAGCAGCAGCAGCAACAGCCGCCACCGCCGCCGCCGCCGCCGCCGCCTCCTCAGCTTCCTCAGCCGC
CGCG
$\begin{array}{ll}\text { HD chimeric primer F: } & \text { 5' 6-FAM-ATG AAG GCC TTC GAG TCC CTC AAG TCC 3' } \\ \text { HD chimeric primer R: } & \text { 5' CGG TGG CGG CTG TTG CTG CTG CTG CTG CTG_3' }\end{array}$

Figure 3 Schematic representation of Huntington disease (HD) chimeric assay. (a) HD chimeric assay primers. (b) HD chimeric primer sequences. 

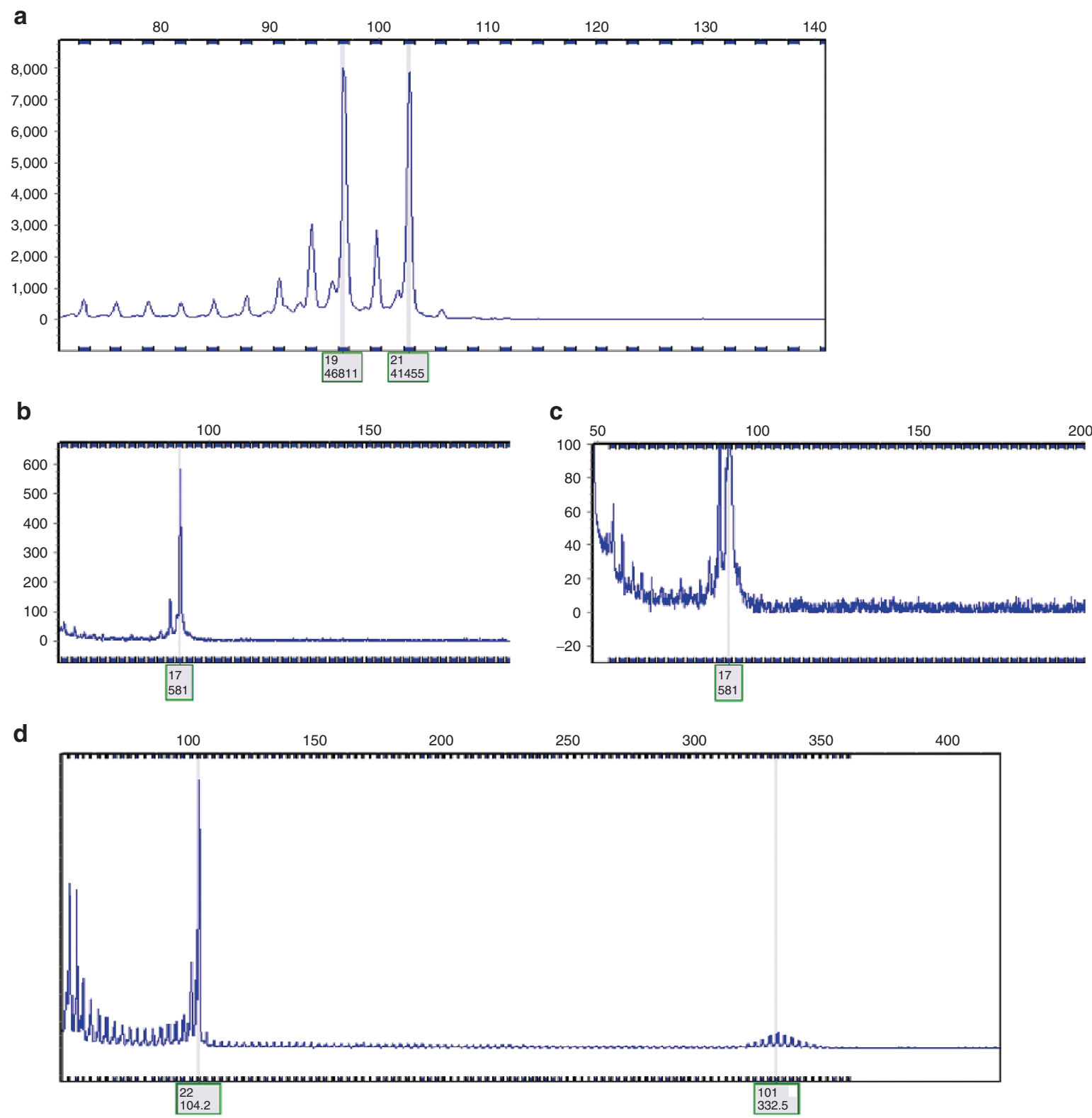

Figure 4 Triplet-repeat primed polymerase chain reaction (PCR) results with different allele sizes. (a) Triplet-repeat primed PCR results with two normal alleles (19 and 21 CAG repeats). There is a stark decrease in fluorescence after the larger allele peak, and then a flat line to the end of the electropherogram. (b) Triplet-repeat primed PCR results with 17/17 homozygous. (c) 17/17 homozygous zoomed in to show the flat line after the 17 allele peak. (d) Triplet-repeat primed PCR results with a normal allele of 22 CAG repeats and a pathogenic allele of 101 CAG repeats. Note the stuttering continues from the smaller to the larger allele and then decreases to a flat line after the 101 CAG repeat.

It is recommended that all laboratories consider the use of either Southern blot or TP PCR or establish a formal referral arrangement with a facility that offers this testing for those cases requiring genotypic resolution.

\section{Interpretations}

Since 1998, it has been the intent of the CAP/ACMG Biochemical and Molecular Genetics Resource Committee to standardize the accuracy of CAG repeat quantitation. Based on the analysis of previous $\mathrm{CAP} / \mathrm{ACMG}$ proficiency testing survey results for $\mathrm{HD}$, the acceptable range for sizing CAG repeats was revised in 2012. ${ }^{34}$ Acknowledging the technical limitations of size analysis, the
ACMG supports the following acceptable ranges for HD clinical testing and as grading criteria for the CAP/ACMG proficiency testing survey: consensus size \pm 2 repeats for alleles with less than 50 repeats; consensus size \pm 3 repeats for alleles with $50-75$ repeats; and consensus size \pm 4 repeats for alleles with $>75$ repeats.

Reporting clinical results. Elements considered essential to the reporting of clinical test results are described in detail in ACMG's Standards and Guidelines for Clinical Genetics Laboratories. The following additional elements should also be included in the reporting of an HD genotype.

The methodology used to assign the genotype. If PCR methodology was used, then a description of the primer 
pair(s) should be included as well as the method of amplicon separation and detection. Furthermore, each report should contain a statement regarding PCR sensitivity for the detection of large expansions. If a Southern blot was required, then the restriction enzyme(s) and probe(s) should be identified. Each report must state the CAG repeat length categories and descriptors currently utilized in clinical practice, and each reportable genotype should be classified and interpreted using these categorical definitions.

CAG repeat number. Each report must include the CAG repeat numbers of both alleles with the precision of sizing fulfilling the criteria recommended by the CAP/ACMG Biochemical and Molecular Genetics Resource Committee. For large alleles determined by PCR, Southern blot, and/or TP PCR, qualifying terms such as "approximately" or "estimated" can be used but should not be written because these could create unnecessary interpretive ambiguity. All positive results should state that genetic counseling is indicated and that testing is available for other at-risk family members.

The following points regarding alternative diagnoses may be included:

- HD phenocopies represent $23 \%$ of patients referred for molecular confirmation of a clinical diagnosis of $\mathrm{HD},{ }^{35,36}$ and several other loci (HDL1 and HDL2) have been identified. ${ }^{37,38}$ As such, HTT gene expansion in these patients will be negative. ${ }^{39}$ Furthermore, the considerable clinical overlap between HD and dentatorubral-pallidoluysian atrophy may warrant a recommendation of ATN1 gene testing in those patients referred with a clinical diagnosis of $\mathrm{HD}$ who subsequently test negative for the expansion. ${ }^{40}$

- Comments on the significance of mutable normal alleles and HD alleles with reduced penetrance should not be overinterpreted in the absence of specific and well-documented clinical and/or family histories. Laboratories should be familiar with the published data regarding these CAG repeat length descriptors and should be able to articulate an interpretation that balances these data with the specific patient/pedigree information provided to the diagnostic laboratory at the time of testing. ${ }^{11-14,34,40,41}$

\section{ACKNOWLEDGMENT}

This guideline was approved by the ACMG Board of Directors on 31 July 2014.

\section{DISCLOSURE}

The authors direct laboratories that offer clinical molecular genetic testing for Huntington disease.

\section{REFERENCES}

1. Potter NT, Spector EB, Prior TW. Technical standards and guidelines for Huntington disease testing. Genet Med 2004;6:61-65.

2. Bates GP, Harper P, Jones L. Huntington's Disease. Oxford University Press: New York, 2002.
3. Aziz NA, van Belzen MJ, Coops ID, Belfroid RD, Roos RA. Parent-of-origin differences of mutant HTT CAG repeat instability in Huntington's disease. Eur J Med Genet 2011;54:e413-e418.

4. Zheng Z, Diamond MI. Huntington disease and the huntingtin protein. Prog Mol Biol Trans/ Sci 2012;107:189-214.

5. Losekoot M, van Belzen MJ, Seneca S, Bauer P, Stenhouse SA, Barton DE; European Molecular Genetic Quality Network (EMQN). EMQN/CMGS best practice guidelines for the molecular genetic testing of Huntington disease. Eur J Hum Genet 2013:21:480-486.

6. Kremer B, Goldberg P, Andrew SE, et al. A worldwide study of the Huntington's disease mutation. The sensitivity and specificity of measuring CAG repeats. N Engl J Med 1994;330:1401-1406.

7. Nance MA, Seltzer W, Ashizawa T, et al. ACMG/ASHG Statement. Laboratory guidelines for Huntington disease genetic testing. Am J Hum Genet 1998;62:1243-1247.

8. Kelly TE, Allinson P, McGlennen RC, Baker J, Bao Y. Expansion of a 27 CAG repeat allele into a symptomatic huntington disease-producing allele. Am J Med Genet 1999;87:91-92

9. Myers RH, MacDonald ME, Koroshetz WJ, et al. De novo expansion of a (CAG) $n$ repeat in sporadic Huntington's disease. Nat Genet 1993;5:168-173.

10. Goldberg YP, Kremer B, Andrew SE, et al. Molecular analysis of new mutations for Huntington's disease: intermediate alleles and sex of origin effects. Nat Genet 1993:5:174-179.

11. Goldberg YP, McMurray CT, Zeisler J, et al. Increased instability of intermediate alleles in families with sporadic Huntington disease compared to similar sized intermediate alleles in the general population. Hum Mol Genet 1995:4:1911-1918.

12. Chong SS, Almqvist $E$, Telenius $H$, et al. Contribution of DNA sequence and CAG size to mutation frequencies of intermediate alleles for Huntington disease: evidence from single sperm analyses. Hum Mol Genet 1997;6: 301-309.

13. Rubinsztein DC, Leggo J, Coles $R$, et al. Phenotypic characterization of individuals with 30-40 CAG repeats in the Huntington disease (HD) gene reveals HD cases with 36 repeats and apparently normal elderly individuals with 36-39 repeats. Am J Hum Genet 1996;59:16-22.

14. Brinkman RR, Mezei MM, Theilmann J, Almqvist E, Hayden MR. The likelihood of being affected with Huntington disease by a particular age, for a specific CAG size. Am J Hum Genet 1997;60:1202-1210.

15. Nance MA, Mathias-Hagen V, Breningstall G, Wick MJ, McGlennen RC. Analysis of a very large trinucleotide repeat in a patient with juvenile Huntington's disease. Neurology 1999;52:392-394.

16. Telenius H, Kremer B, Goldberg YP, et al. Somatic and gonadal mosaicism of the Huntington disease gene CAG repeat in brain and sperm. Nat Genet 1994;6:409-414.

17. American College of Medical Genetics. Standards and Guidelines for Clinical Genetics Laboratories, 3rd edition. American College of Medical Genetics: Bethesda, MD 2002

18. The Huntington's Disease Collaborative Research Group. A novel gene containing a trinucleotide repeat that is expanded and unstable on Huntington's disease chromosomes. Cell 1993;72:971-983.

19. Riess O, Noerremoelle A, Soerensen SA, Epplen JT. Improved PCR conditions for the stretch of (CAG)n repeats causing Huntington's disease. Hum Mol Genet 1993:2:1523.

20. Valdes JM, Tagle DA, Elmer LW, Collins FS. A simple non-radioactive method for diagnosis of Huntington's disease. Hum Mol Genet 1993;2:633-634.

21. Warner JP, Barron LH, Brock DJ. A new polymerase chain reaction (PCR) assay for the trinucleotide repeat that is unstable and expanded on Huntington's disease chromosomes. Mol Cell Probes 1993;7:235-239.

22. Andrew SE, Goldberg YP, Theilmann J, Zeisler J, Hayden MR. A CCG repeat polymorphism adjacent to the CAG repeat in the Huntington disease gene: implications for diagnostic accuracy and predictive testing. Hum Mol Genet 1994;3:65-67.

23. Le H, Fung D, Trent RJ. Applications of capillary electrophoresis in DNA mutation analysis of genetic disorders. MP, Mol Pathol 1997;50:261-265.

24. Williams LC, Hegde MR, Herrera G, Stapleton PM, Love DR. Comparative semiautomated analysis of (CAG) repeats in the Huntington disease gene: use of internal standards. Mol Cell Probes 1999;13:283-289.

25. Bruland O, Almqvist EW, Goldberg YP, Boman H, Hayden MR, Knappskog PM. Accurate determination of the number of CAG repeats in the Huntington disease gene using a sequence-specific internal DNA standard. Clin Genet 1999;55:198-202.

26. Gambardella A, Muglia M, Labate A, et al. Juvenile Huntington's disease presenting as progressive myoclonic epilepsy. Neurology 2001;57:708-711. 
27. Cross G, Pitt T, Sharif A, Bates G, Lehrach H. False-negative result for Huntington's disease mutation. Lancet 1994;343:1232.

28. Gellera C, Meoni C, Castellotti B, et al. Errors in Huntington disease diagnostic test caused by trinucleotide deletion in the IT15 gene. Am J Hum Genet 1996;59:475-477.

29. Margolis RL, Stine OC, Callahan C, et al. Two novel single-base-pair substitutions adjacent to the CAG repeat in the huntington disease gene (IT15): implications for diagnostic testing. Am J Hum Genet 1999;64:323-326.

30. Williams LC, Hegde MR, Nagappan R, et al. Null alleles at the Huntington disease locus: implications for diagnostics and CAG repeat instability. Genet Test 2000;4:55-60.

31. Yu S, Fimmel A, Fung D, Trent RJ. Polymorphisms in the CAG repeat-a source of error in Huntington disease DNA testing. Clin Genet 2000;58:469-472.

32. Guida M, Fenwick RG, Papp AC, Snyder PJ, Sedra M, Prior TW. Southern transfer protocol for confirmation of Huntington disease. Clin Chem 1996;42: $1711-1712$.

33. Jama M, Millson A, Miller CE, Lyon E. Triplet repeat primed PCR simplifies testing for Huntington disease. J Mol Diagn 2013;15:255-262.

34. Palomaki GE, Richards CS. Assessing the analytic validity of molecular testing for Huntington disease using data from an external proficiency testing survey. Genet Med 2012;14:69-75.
35. Rosenblatt A, Ranen NG, Rubinsztein DC, et al. Patients with features similar to Huntington's disease, without CAG expansion in huntingtin. Neurology 1998; 51:215-220.

36. Moore RC, Xiang F, Monaghan J, et al. Huntington disease phenocopy is a familial prion disease. Am J Hum Genet 2001;69:1385-1388.

37. Margolis RL, O'Hearn E, Rosenblatt A, et al. A disorder similar to Huntington's disease is associated with a novel CAG repeat expansion. Ann Neurol 2001;50:373-380.

38. Holmes $\mathrm{SE}, \mathrm{O}^{\prime}$ Hearn $\mathrm{E}$, Rosenblatt $\mathrm{A}$, et al. A repeat expansion in the gene encoding junctophilin-3 is associated with Huntington disease-like 2. Nat Genet 2001;29:377-378.

39. Potter NT, Meyer MA, Zimmerman AW, Eisenstadt ML, Anderson IJ. Molecular and clinical findings in a family with dentatorubral-pallidoluysian atrophy. Ann Neurol 1995;37:273-277.

40. Maat-Kievit A, Helderman-van den Enden P, Losekoot M, et al. Using a roster and haplotyping is useful in risk assessment for persons with intermediate and reduced penetrance alleles in Huntington disease. Am J Med Genet 2001;105:737-744.

41. Maat-Kievit A, Losekoot M, Van Den Boer-Van Den Berg H, et al. New problems in testing for Huntington's disease: the issue of intermediate and reduced penetrance alleles. J Med Genet 2001;38:E12. 